\title{
Reflexionen zu gesellschaftlichen Entwicklungen am Beispiel der Interruptio Quo vadis, Gesellschaft?
}

Helge Köhler

Korrespondenz:

Dr. med. Helge Köhler

Zollikerstrasse 193

CH-8008 Zürich

Tel. 0444665465

helge.koehler@triemli.stzh.ch
Die Möglichkeit des medikamenteninduzierten Schwangerschaftsabbruchs und der Wegfall des obligatorischen psychiatrischen Gutachtens vor einer Interruptio haben wahrscheinlich nicht dazu geführt, dass es zu einem starken Anstieg von Schwangerschaftsabbrüchen gekommen ist (Angaben Kanton Zürich). Dennoch sind unerwünschte Schwangerschaften weiterhin ein häufiger Grund für Konsultationen in Praxen und Kliniken. Da nun die ethische Integrität einer Gesellschaft durch die Art und Weise, wie sie mit ihren schutzlosesten Mitgliedern umgeht, präzise abgebildet wird, bedarf diese Tatsache der Reflexion, und zwar sowohl durch die Gesellschaft als auch durch die behandelnden Ärztinnen und Ärzte. Die folgenden Gedanken zu diesem Thema bieten keine Lösungsvorschläge, sie sollen aber dieses wichtige gesellschaftliche und medizinische Anliegen von verschiedenen Seiten beleuchten und den Umgang mit dem Problem ungewollter Schwangerschaften wieder mehr in das Bewusstsein aller Beteiligten rücken.

Vor jedem Schwangerschaftsabbruch findet ein ärztliches Behandlungsgespräch statt. Die Intention dieses Kontaktes zwischen Patientin und Ärztin/Arzt sollte ursprünglich nicht nur in der Feststellung der Schwangerschaft und des Gestationsalters sowie in der Aufklärung über Behandlungsschritte liegen, sondern der wichtigste Grund dieses Gesprächs war doch das Ausloten gangbarer Alternativen zur Interruptio. In der täglichen Praxis kommt es aber häufig zu einer Verschiebung der Gesprächsintention. De facto spielt die Suche nach Auswegen aus dem Schwangerschaftskonflikt nur eine untergeordnete Rolle. Das Beratungsgespräch wird von den Frauen (Paaren) nicht als verpflichtendes Suchen nach Alternativen zum Abbruch wahrgenommen, sondern vielmehr lediglich als Aufklärungsgespräch für den medizinischen Eingriff (Operation, medikamentöser Abbruch) verstanden. Deshalb ist der Wechsel der Einstellung von «gegen die Schwangerschaft» zu «für die Schwangerschaft» im Rahmen des Beratungsgesprächs ein seltenes Ereignis. Der Schwangerschaftsabbruch wird somit in den Augen der Konsultierenden mehr als selbstverständliche Pflichtleistung des Gesundheitssystems und weniger als Notmassnahme in einer verzweifelten Situation

\section{Interruption de grossesse: où va la société?}

La primauté de tout acte médical est la promotion et la protection de la vie de la personne humaine et de sa santé. Traiter des femmes en situation de grossesse non désirée aboutit forcément à une situation conflictuelle qui entraîne de graves conséquences. Bien que le présent exposé ne puisse amener de solution idéale, il vise à replacer le thème de l'interruption de grossesse au centre d'un large débat. En tant que médecins, femmes et hommes, nous y sommes tenus.

angesehen. Die Hemmschwelle, diesen einschneidenden Eingriff durchführen zu lassen, ist oft sehr niedrig. Das Recht auf Selbstbestimmung überwiegt die Pflicht zum Schutz von Leben. Falls diese Entwicklung andauert, ist es denkbar, dass in Zukunft jede Schwangere über die Möglichkeit eines Abbruchs aufgeklärt werden muss und dass der Arzt bei Unterlassung dieser Aufklärung für etwaige Komplikationen aufgrund der Schwangerschaft (körperlich, seelisch, finanziell usw.) zur Rechenschaft gezogen werden kann.

In der gynäkologisch-geburtshilflichen Facharztausbildung ist es gängig, die ersten operativen Erfahrungen an Interruptiones zu machen. Es sind im allgemeinen die jüngsten und unerfahrensten Ärztinnen und Ärzte, die diese Eingriffe durchführen. Noch bevor die Fähigkeit zu grundlegenden Untersuchungsmethoden, zum Beispiel zur korrekten Abnahme eines Pap-Abstriches, erworben wird, erwartet man von den jungen Kolleginnen und Kollegen die Beherrschung eines so komplexen und heiklen Gebietes wie der Beratung und Behandlung von Frauen mit unerwünschter Schwangerschaft.

Neben diesem Missstand fällt nun zusätzlich auf, dass einerseits der von der Gesellschaft akzeptierte und vom Gesundheitssystem praktizierte nachvollziehbare hohe Aufwand zur Verlängerung der letzten Lebensspanne eines sehr 
alten oder sehr kranken Menschen und andererseits die Bereitschaft zur Beendigung eines ungeborenen Lebens in einem krassen Widerspruch stehen.

Wenn man sich mit dem Thema Schwangerschaftsabbruch auseinandersetzt, kommt man schnell zu der Erkenntnis, dass es den in der Medizin so geliebten Goldstandard hier nicht geben kann. Das wertvolle Recht der Schwangeren auf Selbstbestimmung einerseits und die Verantwortung für das ungeborene Leben andererseits stehen sich bei Schwangerschaftskonflikten diametral gegenüber. Dogmatische Lösungsmuster sind fehl am Platz. Aber als Ärztinnen und Ärzte müssen wir lernen, die Verunsicherung und Irritation einer Frau durch die Diagnose einer Schwangerschaft auszuhalten, und wir müssen uns davor hüten, diesen Gemütszustand prima vista als «negatives Desiderium» fehlzuinterpretieren. Die wünschenswerte Empathie in dieser Situation darf nicht voreilig in einen Schwangerschaftsabbruch münden. Die Priorität im Rahmen der Beratung muss klar im Erhalt der Schwangerschaft liegen. Eine Interruptio darf weder ein notwendiges Übel bei der Karriereplanung sein noch als Therapeutikum für das Gefühl der Überforderung dienstbar gemacht werden. Der Schwangerschaftsabbruch kann nur die Ultima ratio sein. Dazu sind wir Ärztinnen und Ärzte aber dringend auf ein schwangerschaftsförderndes Klima in der Gesellschaft angewiesen. Dies beinhaltet zum einen die Aufwertung des ungeborenen Lebens (der Wegfall des obligatorischen psychiatrischen Gutachtens bewirkte genau das Gegenteil) und zum anderen Massnahmen für ein familienfreundlicheres Leben (bezahlbare Kinderbetreuung, mehr Teilzeitstellen, finanzielle Wertschätzung der Erziehungsarbeit). Da Kinder doch die wichtigste Zukunftsinvestition sind, ist die gesellschaftliche Akzeptanz von massiven Defiziten in diesem Bereich nicht nachvollziehbar. Auch notwendig sind breitangelegte Aufklärungskampagnen über die Folgen von ungeschütztem Sex, ähnlich wie es bei der Aidsprävention oder auf anderem Gebiet bei der Aufklärung über die Gefahren von Alkohol im Strassenverkehr geschieht.

Es ist gut, dass es die Möglichkeit des straffreien und risikoarmen Schwangerschaftsabbruchs gibt, und es ist richtig, dass die Entscheidungskompetenz über einen Abbruch ausschliesslich bei der betroffenen Frau liegt, aber dieser Eingriff ist, obwohl technisch einfach, doch einschneidend und schwerwiegend. Deshalb darf ein Schwangerschaftsabbruch nur als letzte Behandlungsmöglichkeit einer sonst ausweglosen Situation nach Überprüfung aller möglichen Alternativen verstanden werden, einschliesslich der Möglichkeit, das Neugeborene zur Adoption freizugeben, wenn ein Leben mit dem Kind als unmöglich angesehen wird. Es ist unbedingt notwendig, dass diese Einstellung fest in das Bewusstsein aller Beteiligten (Patientinnen, Gesetzgeber, Ärztinnen und Ärzte, soziale Dienste usw.) installiert wird. Es darf nicht sein, dass das Ausmass der Sensibilität für die Rechte einer «Personengruppe» von deren Fähigkeit, diese Rechte zu artikulieren, abhängig ist. Das ungeborene Leben muss als schützenswert angesehen werden.

Diese kritische Sichtweise bezüglich der Vorgehensweise bei ungewollter Schwangerschaft resultiert weder aus einer radikal-religiösen Einstellung noch aus einem patriarchalischen Gesellschaftsverständnis. Die stark emotionalisierte Art und Weise der Argumentation von Gegnern und Befürwortern der Interruptio kann dem zu beurteilenden Einzelfall nicht gerecht werden, sondern das Thema «Schwangerschaftsabbruch» wird hier von einigen Gruppierungen als Argumentationswerkzeug für andere Intentionen missbraucht. Keinesfalls sind diese Gedanken dazu geeignet, die individuelle Vorgehensweise zu beurteilen, geschweige denn sie zu verurteilen. Aber die Gesellschaft hat die Pflicht, einen Bereich, in dem es um die sensible und heikle Abwägung von Rechtsgütern geht, immer wieder zum Thema einer breitangelegten, sachlichen Diskussion zu machen. 